\title{
A Study of Data Classification for Hybrid Storage System Based on Genetic Algorithm
}

\author{
Chunmin Qiu, Jie Shan \\ Binzhou Polytechnic, Binzhou, Shandong Province, China \\ bzqcm@163.com, shanjie828@163.com
}

\begin{abstract}
The explosive growth in data storage system so that people demand for large capacity and fast access to the increasingly urgent. At present, able to bear this responsibility is the rapidly growing solid state disk (SSD) and the traditional hard disk drive (HDD). The capacity of HDD is large, but its random reading speed may not have greatly improved. The random reading speed of SSD is a hundred of times faster than HDD, but its ectopic updating, limited number of erase, and expensive price had restricted its popularization. In recent years, the development of hybrid storage system consisting of SSD and HDD was rapid. In order to raise Capability/price Ratio of the hybrid storage system, we must classify the storage of data according to the characteristics of SSD and HDD. This paper presented a data classification method based on genetic algorithm, It gave full play to the advantages of the rapid random reading speed of SSD, the large capacity and in situ update of HDD in accessing a large amount of data in hybrid storage system, and it restrained the inferiority of limited number of erase on SSD and slower random reading speed on HDD.
\end{abstract}

Index Terms - Hybrid storage, Data classification, File heat, Genetic algorithm

\section{Introduction}

With the rapid development of computer technology and Internet technology, we face the explosive growth of data. We always wish to continuously increase the storage capacity, reduce the manufacturing cost, and improve the storage performance of the storage device. Large capacity storage devices currently used are mainly HDD and SSD

HDD access the data by rotating the head, the faster the speed of rotation, the faster data access. After more than half a century of development, the rotating speed of HDD had promoted from 3600RPM to $15000 \mathrm{RPM}$. At present, 7200RPM rotating speed HDD is the mainstream product. With the up of HDD head rotation speed, power consumption is also increasing, and the rotation speed may not have greatly improved.

In 1989, the world's first SSD was produced. After more than 20 years of development, a hundred times the access speed of SSD over HDD, and the power consumption is very small. SSD without any mechanical part, has excellent seismic performance, and more and more small volume. If there is any storage medium to replace HDD used to access the data in computer system, SSD is only[1].

Compared with the hard disk, SSD random read faster, file fragmentation has no effect on reading speed; SSD disk writing speed is slower than the speed of reading, and updating data does not like HDD as the original data rewriting, but first the original data set to an invalid, and then write new data to free pages; SSD recovery storage space by erasing the invalid data block. But SSD storage erasing times of block is limited, usually ten thousand to one hundred thousand times, so the frequently updated data should not be stored in solid state disk The price of SSD is more expensive than HDD, storage capacity is relatively small, had restricted the popularization and application of SSD[2].

The storage system users, of course, hope with larger capacity, lower cost, higher performance, but whether it is the hard disk or solid state disk can not set the three big advantages in one. More and more researchers use HDD with large capacity and SSD with fast reading speed and smaller capacity building hybrid storage system, in order to achieve the user's that three expectations for storage system. Current research focus is how to classify data in hybrid storage system, store the high frequency reading data and low frequency updating data in the solid state drive, and can dynamicly adjustment, in order to raise Capability/price Ratio of the hybrid storage system[3].

This paper presents a data classification method based on genetic algorithm according to the characteristics of SSD and HDD. It gave full play to the advantages of the rapid random reading speed of SSD, the large capacity and in situ update of HDD in accessing a large amount of data in hybrid storage system, and it restrained the inferiority of limited number of erase on SSD and slower random reading speed on HDD.

\section{Related Work}

A. Operational principle of hybrid storage system

Modern computer still follow the stored program principle that was proposed by von Neumann, the data must be loaded into internal memory, in order to be dealt with by CPU, Updated data must be stored in the external memory, so that it can be permanently retained. Because the random reading speed of HDD is much slower than writing speed of internal memory, external storage system only be consisted of HDD restricts the overall performance of the computer system upgrade. The hybrid storage system consisting of SSD and HDD can greatly improve the reading speed of the external memory.

In the hybrid storage system, according to the physical properties of SSD, try to store the data read high frequently in SSD. When an application program is going to process data, first of all to read the data from SSD, if hit written into the memory, if miss, to read the data from HDD; When the application program save data to the external memory, the 
updated data is written to HDD, if there is the original data in SSD, is set to invalid; At the end of the application, the update data stored in HDD, the original data of which is in SSD, is migrated to SSD [4]. Data reading and writing process is as shown in Fig.1.

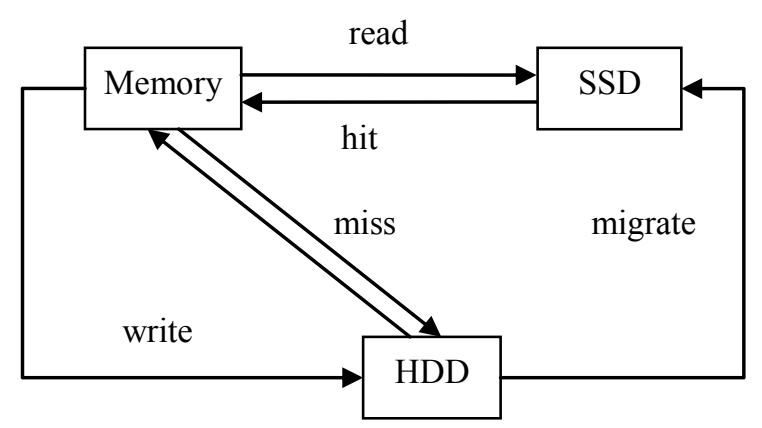

Fig. 1 Hybrid storage system data reading and writing process chart

\section{B. Data distribution in hybrid storage system}

Hybrid storage, in fact, is a kind of data storage method, it took the advantage of SSD high random reading and HDD high order writing, avoids SSD storage blocks of frequent erasing and large number of random reading operation on hard disk, foster strengths and circumvent weaknesses, greatly raise Capability/price Ratio of the storage medium.

Obviously, if want to play to the performance of the hybrid storage system, must be classifying data, one kind is frequently accessed data, called heat data, one kind is not accessed data for a long time, is called cold data. Industry research and analysis shows that in most of the storage system, $70 \% \sim 80 \%$ of the data is cold[5]. The heat data should be stored in SSD, cold data stored on HDD. Data classification of hot and cold is the current hybrid storage research hot spot.

The smallest unit of storage data is the data block, a data file has several data blocks. On the data of hot and cold test can block as the unit, also can file for the unit. Data block level heat data detection is relatively fine, hot and cold on data files for break up, but the data of this grain size testing needs a large amount of data block level metadata to support, increasing the overhead storage system, for large data files, especially. Large data files, such as commodity sales record, 1 to 2 months sales record recently may be frequently accessed, records of the other months data becomes cold, if the entire file as hot data stored in SSD, most of these data may not be visited, will be wasted valuable system resources. Distinguish file of heat and cold so needs to consider the history of file access frequency, file size, number of users, and the size of SSD capacity, and other comprehensive factors[6].

\section{Data Classification Algorithm Design}

In the light of the hybrid storage system consisting of SSD and HDD, speaking, reading and writing characteristics, puts forward a calculation model of the heat data in file as a unit, and genetic algorithm was applied to classification file for hot and cold.

\section{A. File heat}

1) File creation heat: Definition of file i creation heat is

$$
H N_{\mathrm{i}}=\frac{1}{T N_{i}}
$$

where $T N_{i}$ indicates file i creation time the length of time up to now. Recently the new file to be accessed the probability of large, high heat.

2) File reading heat: Definition of file i reading heat is

$$
H R_{i}=\left\{\begin{array}{cc}
0 & \text { Filenot been read } \\
\sum_{j=1}^{m} \frac{\beta_{j}}{T R_{i j}} & \text { File been read m times }
\end{array}\right.
$$

where $T R_{i j}$ indicates file $\mathrm{i} \mathrm{j}$-th reading time the length of time up to now[7]. In order to more accurately reflect the amount of data read by the document reading each heat effects , the coefficient of heat introduced $\beta_{j}$, Its value is the ratio of the amount of $j$-th reading data and file size.

3) File updating heat: Definition of file i updating heat is

$$
H W_{i}=\left\{\begin{array}{cc}
0 & \text { File not been updated } \\
\sum_{j=1}^{k} \frac{1}{T W_{i j}} & \text { File been updated k times }
\end{array}\right.
$$

where $T W_{i j}$ indicates file $\mathrm{i} \mathrm{j}$-th updating time the length of time up to now.

4) File space heat: Definition of average reading-writing heat for all files in storage system is

$$
H A=\frac{1}{n} \sum_{i=1}^{n} \frac{H R_{i}+H W_{i}}{m+k}
$$

where $\mathrm{m}$ is the number of times file $\mathrm{i}$ is read, $\mathrm{k}$ is the number of times file $\mathrm{i}$ is updated. If two files with the same heat, the Larger space occupation file should be stored in SSD, SSD can play so random read speed advantage. Definition of file i space heat is

$$
H S_{i}=\frac{S_{i} \times H A}{S}
$$

where $S_{i}$ indicates file-occupying-space of file i, $S$ indicates the total file-occupying-space of all files stored in the storage system. heat is

5) File attention heat: Definition of file i file attention

$$
H U_{i}=l \times H A
$$

where $l$ is the number of users to access file $\mathrm{i}$. 
6) File heat: It's the total of the above 5. So definition of file $i$ heat is

$$
H_{i}=H N_{i}+H R_{i}+H W_{i}+H S_{i}+H U_{i}
$$

\section{B. Application of genetic algorithm}

Through the genetic algorithm to find the best combination of files, it's total heat is the highest in all of the files-combination SSD can store. The chromosome of individuality within population is encoded using a binary number, chromosome $\mathrm{i}$ is

$$
X_{i}=\left(x_{i 1}, x_{i 2}, \ldots, x_{i n}\right)
$$

indicates a combination of files, where $\mathrm{n}$ is total number of files, $x_{i k}(1 \leq k \leq n)$ equals 0 or 1 , if it equals 1 , said file $\mathrm{k}$ belongs to files-combination $\mathrm{i}$. The total heat of $X_{i}$ is

$$
H_{X_{i}}=\sum_{k=1}^{n} x_{i k} H_{k}
$$

Population size $\mathrm{N}$ is randomly generated, $10 \leq N \leq 160$, is 10 multiples. $\mathrm{N}$ randomly selected from all files-combinations form the initial population[8].

The fitness function is designed for

$$
\text { Fit }(y)=\sqrt{\frac{y-b}{10 a}}
$$

Where, $a$ and $b$ are recalculated in each generation,

$$
\begin{aligned}
& b=H_{\text {min }} \\
& a=H_{\text {max }}-H_{\text {min }} \\
& H_{\text {min }}=\min \left\{H_{X_{1}}, H_{X_{2}}, \ldots, H_{X_{N}}\right\} \\
& H_{\max }=\max \left\{H_{X_{1}}, H_{X_{2}}, \ldots, H_{X_{N}}\right\}
\end{aligned}
$$

Obviously

$$
\begin{aligned}
& y \in\left[H_{\text {min }}, H_{\text {max }}\right] \\
& \text { Fit }(y) \geq 0 \\
& \text { Fit }\left(H_{\text {min }}\right)=0 \\
& \text { Fit }\left(H_{\text {max }}\right)=1 \\
& \text { Fit }(y) \in[0,1]
\end{aligned}
$$

Function curve is shown in Fig. 2, as to meet the requirements of the design of fitness function[9].

In the selection operation, the first, files-combinations, whose occupying-space is greater than the effective capacity of SSD, are eliminated. And then, copy the files-combination to the next generation, whose heat is the highest. Finally, using the roulette algorithm, select $\mathrm{N}-1$ files-combinations. The probability of crossover operation is set to $P_{\text {cross }}=0.5$, this will not only make the files-combinations within one generation have no more than $50 \%$ of the possible crossover

\begin{tabular}{|c|c|}
\hline 1 & Initialize parameter: $\mathrm{N}=\operatorname{rand}(10,160), P_{\text {cross }}=0.5, P_{\mathrm{var}}=0.01$ \\
\hline 2 & Randomly generate initial population $\mathrm{PP}=\left\{X_{1}, X_{2}, \ldots, X_{N}\right\}$ \\
\hline 3 & $\begin{array}{l}\text { If the population PP meet the termination condition } V_{F i t}<10^{-4} \text {, } \\
\text { then output the best files-combination }\end{array}$ \\
\hline 4 & $\begin{array}{l}\text { Else, calculate the fitness values of the individuals in the population } \\
\text { PP }\end{array}$ \\
\hline 5 & Perform selection operation on PP using the algorithm of roulette \\
\hline 6 & $\begin{array}{l}\text { Perform random crossover operation on } \mathrm{PP} \text { according to the } \\
\text { crossover probability }\end{array}$ \\
\hline 7 & $\begin{array}{l}\text { Perform random variation operation on PP according to the variation } \\
\text { probability }\end{array}$ \\
\hline 8 & Judge new population PP whether meeting termination condition \\
\hline
\end{tabular}
operation, does not destroy files-combination with high fitness value, but also enable the search state of genetic algorithm to keep sensitive. The probability of variation operation is set to $P_{\text {var }}=0.01$, This not only ensure the algorithm can expand the search ability, avoid falling into local maximum values, and can limit the randomness of the algorithm[10]. Algorithm termination condition is the variance $\left(V_{F i t}\right)$ of individual fitness of the population is less than $10^{-4}$.

The algorithm is shown in TABLE I.

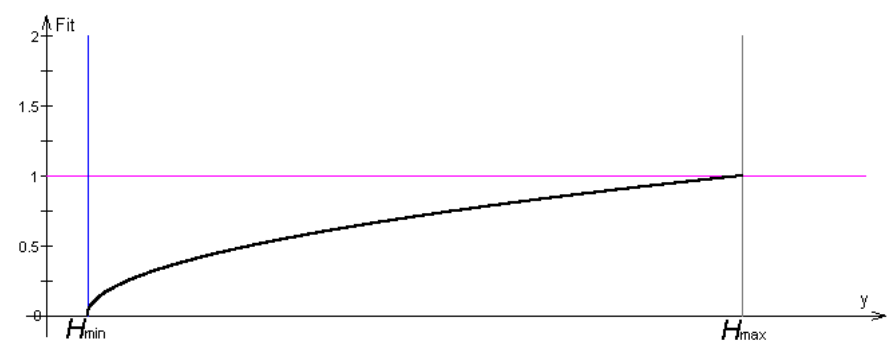

Fig. 2 Fitness function curve chart

TABLE I Genetic algorithm

\section{Summary}

By analyzing the characteristics of the hybrid storage systems composed of SSD and HDD, Aiming at SSD rapid random access, ectopic update, erasing times limited,and aiming at HDD rapid sequential writing speed, unlimited updating times, this paper presented the data distribution patterns for frequently-read data stored in SSD, classified the data in file as a unit, built mathematical model for file heat. Used genetic algorithm to search files-combination which has the highest heat and meet the SSD storage constraints. Practice had proved that the algorithm convergence speed faster, could help hybrid storage system play their own advantages, raised Capability/price Ratio of hybrid storage system, and had perfected the solution of hybrid storage system for large data. 


\section{References}

[1] Yan Min, Long Xiao-qi, Zhang Jing, Hou Zhi-chun, He Min, Design and Implement of High Capacity NAND Flash Memory Storage System with Open System Architecture, Microelectronics \& Computer, Vol.26, No.11, pp.13-16, 2009

[2] Yue Lihua, Xiang Xiaoyan, Jin Peiquan, Liu Zhanzhan, An Out Page Logging Approach to Storage Management in Flash-based DBMS, Journal of University of Science and Technology of China, Vol..40, No.5, pp.526-532, 2010

[3] Wang Jiang-Tao, Lai Wen-yu, Meng Xiao-Feng, Flash-Based Database: Studies, Tech-niques and Forecasts, Chinese Journal of Computers, Vol.36, No.8, pp.1549-1567, 2013

[4] Lv Y F, Cui B, He B S, Chen X., Operation-aware Buffer Management in Flash-based Systems, Proceedings of the ACM SIGMOD International Conference, Athens, Greece, pp.13-24, 2011

[5] Mei He, Ling Xing, Guo Li, A Data Migration Strategy for HSM Based on Data Value, Journal of Information \& Computational Science, Vol.8, No.2, pp.832-836,2011
[6] Zhu Qing, Li Xiaoyong, A Review on Hybrid Storage, Microcomputer Applications, Vol.29, No.2, pp.33-38,2013

[7] Song Lina, Dai Huadong, and Ren Yi, Learning Method of Hot-Spot Extent in Multi-Tiered Storage Medium Based on Hume Data Storage File System, Journal of Computer Research and Development, 49(suppl.): 6-11,2012

[8] Grefenstette J J, Optimization of Control Parameters for Genetic Algorithms, IEEE Transaction on Systems, Man, and Cybernetic, Vol.16, No.1, pp.122-128, 1986

[9] Xin Fei and Zhu Aoxin, Research on Fitness Function in Genetic Algorithms, Systems Engineering and Electronics, Vol.20, No.11, pp.58-62,1998

[10] Schafer JD, A Study of Control Parameters Afecting On-line Performance of Genetic Algorithms for Function Optimization, In Proceedings of the Third International Conferenceon Genetic Algorithms(ICGA 3), San Mateo, CA: Morgan Kaufmann Publishers, pp.51-60,1989 